\title{
The Characteristics of Science and Technology Policy Research from the Perspective of the Characteristics of Science and Technology Activity
}

\author{
Zhuo Ren, Wei Song, Xiaobao Peng, Xiaopei Gao \\ School of Public Affairs, University of Science and Technology of China, Hefei, China \\ Email: renzhuo311@126.com, 1162754682@qq.com
}

Received 21 April 2015; accepted 18 June 2015; published 23 June 2015

Copyright $@ 2015$ by authors and Scientific Research Publishing Inc.

This work is licensed under the Creative Commons Attribution International License (CC BY). http://creativecommons.org/licenses/by/4.0/

\section{c) (i) Open Access}

\begin{abstract}
The characteristics of science and technology activities (STA) have important effect on the characteristics of the research of science and technology policy (STP). On this argument, we review the research on the characteristics of STA and the characteristics of the study of STP, discuss the characteristic of STP itself and the effect on the research of STP, therefore summarize the characteristics the research of STP should have. The characteristics of the current STA are as below: its effect is irreversible and of wide range as well as high speed; it faces with the risk of technology and market; it has ethical anomie; it is interdisciplinary and cross level and domain; it has national mission, etc. These characteristics make the research of STP need the following characteristics: Timeliness and scientific; Tolerance and face up to failure; Normative values; Extend research subject; national demand oriented, etc.
\end{abstract}

\section{Keywords}

Science and Technology Activities, Science and Technology Policy, The Research of Policy, Effect

\section{Introduction}

The form and scale of the STA are in constant change with the development of science and technology, and the political, economic, social effect of STA has also been deepened and extended. This, to a certain extent, promotes the complexity of the study of STP, and attracts wide attention of scholars at home and abroad.

For the research of STP, the former research mainly concentrated on the basic concept, research norms and 
the comparison among different countries, etc, only a few researches from the perspective of the characteristics of STA, and few from the perspective of the effect the STA itself has on the STP research. This paper tries to explore the effect the characteristics of STA has on the characteristics of STP research, which is of positive significance to the improvement and development of STP research.

The reason this paper explore the characteristics of STP research from the perspective of the characteristics of STA is that we believe the characteristics of STA to a great extent will influence or determine the characteristics of STP. This is the premise of this paper. The specific reason is that there is a connecting point between STA and STP research-STP. STP is a guide decision that a country or political party makes about the development of science and technology. It is also a basic principle about the direction, objectives, approaches and related measures of science and technology development. In short, STP is the policy about STA. Therefore, STA influences all aspects of STP directly, for example, the object and concrete contents of STP. However, as the object of STP research, STP also has direct influence on STP research. In this way, STP becomes the link point between STA and STP research and the characteristics of the STA indirectly affect the characteristics of STP research because of that conduction function.

In order to achieve the goal of our research, this paper has a literature review about characteristics of STA and STP research. On the basis of that, we exclude the inopportune or inappropriate part and add new features under the background of new era and summarize more complete characteristics of STA, finally infer the requirements that STP should meet.

\section{Literature Review}

According to the principle of "international standardization proposals on the evaluation of STA" from United Nations, STA refers to all the activities that are closely related to the development, production, dissemination and application of the science and technology knowledge in the field of science and technology. It is mainly divided into four categories: research and development test (R \& D), research and experimental development achievement application, science and technology education and training technology services (STS), including application of the achievements of research and other technical services. There is in fact the broad and narrow sense of STP research. The generalized STP research includes all the policy research related to science and technology. But the narrow sense of STP research is science and technology and innovation policy research (STIPS) based on the paradigm of "science—-technology—innovation" [1]. In this paper, we use the generalized sense of STP.

\subsection{The Literature Review about the Characteristics of STA}

The research on the specific characteristics of STA is not too much, mainly concentrated on the research on philosophy of science and technology and science, for example Zhang Zhicang gave a systematic philosophical response about the development of modern science and technology and its related problems [2]. The specific research on the characteristics of STA mainly aimed at the characteristics of STA at that time of the last century, or the characteristics of STA of a local enterprise or university or other countries and regions of modern times, for instance He Yingming made an R\&D statistical investigation on the characteristics of STA in Singapore [3], Yan Gendi, who works in Shanghai Municipal Bureau of statistics, studies the characteristics of science \& technology of large and medium-sized industrial enterprises in Shanghai according to the statistical data of 1998 [4]. The existing study on the characteristics of STA is summarized as follows.

\subsubsection{The Characteristics of STA from the Space Point of View}

From the space point of view, the characteristics are mainly as follows.

The first is the internationalization of the scope of STA which are not confined to a single state. This is mainly benefit from, firstly, the traffic is more and more convenient. There is no traffic barrier for the global STA. Secondly, the development of the Internet makes the whole world into a global village, the interaction between people in different countries has no longer distance. Thirdly, as the consequences of the development of economy and technology, there are many global problems which need to be solved and they provide power for the internationalization of STA [5].

The second is that STA has more and more large-scale, organized, objective researches and development activities in the range of the whole society. There are more and more management agencies, educational institution, research institutes in all kinds of STA. Industry, education and government has formed a separate and comple- 
mentary system of STA. STA are gradually socialized and become an essential factor of society.

At last, the effect of STA is more and more comprehensive, almost in all aspects of social life. That is mainly because that the technology has penetrated into all aspects of social life.

\subsubsection{The Characteristics of STA from the Time Point of View}

From the time point of view, the characteristics are mainly as follows.

Firstly, the role of STA on economic life are non synchronous. The main performance is the lag in time, such as the transformation of scientific and technological achievements into economic benefits needs time interval. But under the push of competition, the lag brought by the interval has been shorter and shorter.

Secondly, the irreversible effect of STA has on society .Once the STA have an effect on the economic and social life, this effect is hard to be revoked. Good effects interact with the development of society, however, the bad one may produce unstable factors to the society.

\subsubsection{The Characteristics of STA from the Perspective of the Subject of STA}

From the perspective of the subject of STA, with the upgrade of the status of science and technology, the improvement of science and technology has become an important national mission. The government more and more involved in the STA. No matter from which perspective, STA are no longer just the career of individuals or institutions, organizations. History has pushed the development of science and technology into national mission.

\subsubsection{The Characteristics of STA from the Perspective of the Form of STA}

From the perspective of the form of STA, the characteristics are as below.

Firstly, in order to achieve the national goal of internal and external policy, there occur many large-scale, multi-levels and multi-field research projects centering on the major technological breakthroughs or long-term plan. This is an important feature of contemporary STA, which shows that STA have been unprecedented systematic [5].

Secondly, the form of STA has been more and more diversified and it affects the politics, economy, society in many ways.

\subsection{The Literature Review on the Characteristics of STP Research}

Most of the existing literature on science and technology policy research are from the perspective of the characteristics of the times and the characteristics of research object, but few comes from the perspective of the characteristics of science and technology activities.

\subsubsection{The Characteristics of STP Research from the Their Own Scale Characteristics and the Lack in the Research}

Sheng Jianxin and Cheng Liangbin believe that the characteristics of contemporary STP research are: increasing scale; comprehensive and specialized research; more comparative research method which are used. These three characteristics are summarized from the perspective of the scale, the content and the method of STP research [6]. Some scholars summed up the lack of STP research of China, mainly as follows: STP research is not clearly defined; it is scattered, has not formed the core authors, lack theory and method basis and doesn't have a readymade research paradigm; there is a gap between the theory and the practice of STP [7].

In general, the research above on the characteristics of STP research is mainly from their own scale characteristics and the lack in the research, without taking into account the characteristics of the STA.

\subsubsection{The Characteristics of STP Research from the Perspective of the Form of STP Research}

From the perspective of the form of STP research, the "big science" era requires rapid development of STP research at the aspect of the national scale and international cooperation [8]. The characteristic of the "big science era" has a direct impact on the trend of the scale and internationalization of STA. This requires that STP research must be a national and international collaborative study, which is no longer alone behind closed doors. In this regard, Luan Chunjuan and other scholars do some macro and medium econometric analysis from the national level and organization level and decide the cooperation network of high yield state and organization on international STP research [9]. 
STP Research is also systematic and the integrate [5]. Science and technology exist in the various fields of society, and the exploration of science and technology research continues to extend in the macroscopic and the microscopic aspects. The frontiers of science continue to refine and various disciplines constantly blend. The characteristics of STA cause the systematicness of the STA research continuously, and cover all aspects of social life, having a certain degree of integrity.

\subsubsection{The Characteristics of STP Research of Other Countries}

In addition, some scholars study the characteristics of STP research of other countries. This paper holds that the study has certain reference value for the study of the characteristics of Chinese STP. For example, there are some scholars studying the characteristics of USA STP as follows: demand oriented, for decision-making; precise quantification and trying to form a collaborative development in the aspect of theoretical framework, the model and the data; the scientific community construction, the reason lies in the study of the sociology of science shows, science progress depends on the interaction between the scientists who have the same or similar research problems and research methods in the scientific community [10].

\section{The Characteristics of STA}

This paper is based on the literature review and the current characteristics of the times. The characteristics of STA of this era are refined and summarized as follows.

\subsection{Effect of STA: Wide Range, High Speed and Irreversible}

Globalization has reached a very mature stage, this causes the effect of STA which needs the whole world to enjoy or assume. In the promotion of competition, the transformation from science and technology into the economic and social benefits has become faster and faster. Therefore, STA has a very fast effect to the whole country and even the whole world. Not only that, the effect of science and technology on society is also irreversible, which needs to be treated with caution. The best example of the impact of the development of science and technology on the present life is network. According to a survey online, Internet users less than 8 hours accounted for only $19.6 \%$ of the respondents, and in 12 hours or more accounted for $19.5 \%$. Different Culture, ideas on the network blend or conflict. Network provides a wealth of information to people, at the same time it also brings some negative inevitable factors, such as the existence of the network garbage. What's more, negative influence on teenagers is particularly serious. It may influence the formation of the correct outlook on life for young people.

From the reasons presented above, the characteristic of STA in time and space have a great impact on human society.

\subsection{STA Is Facing the Risk of Technology and Market etc.}

The STA is faced with certain risk; the so-called risk refers to the probability estimation of unacceptable consequences, including technical risk and market risk.

Technology risk refers to the various technical problems related with new technology or emerging technologies. There are many causes, such as technical complexity is too high, the cross technology is too strong. These risks are likely to cause that technology cannot be successfully developed or miss the market opportunity. Market risk refers to the risk when science and technology goes into the market, which can be divided into the available of various elements of the value chain, the difference of product and the acceptability of market. For instance, the complexity of the technology allows high cost for buyers and sellers reaching agreement on the product performance. That is high transaction costs. The research of the domestic scholar think that the success rate of high-tech enterprise $\mathrm{R} \& \mathrm{D}$ project is only $60 \%$, the possibility of successful access into the market is only $30 \%$, while that of high-tech products is only $12 \%$ [11]. These risks are important causes of the failure of the STA [12].

\subsection{Ethical Anomie}

STA sometimes ignore the ethics due to the pursuit of the interests, which leads to the problem of ethical anomie. Sometimes it cannot get a good trade-off in other social and ethical issues. STA profoundly change the relationship between man and nature, man and society and man and man's, bring hitherto unknown ethical problems to 
the human society. The ethical anomie caused by high-tech has brought huge impact to the original human ethics and ethical risk and the further development of high-tech. For example, cloning technology has the vital significance on improvement of human life science research, but brings subversive impact on the basic human ethics and bioethics.

\subsection{STA: Cross Discipline, Cross Level, Cross Domain}

STA not only have development to the depth in the vertical direction, but also have intersection, integration in the horizontal direction continuously. There is no clear division between the various disciplines who learn from each other. Each level also crosses each other and complements each other. Various fields also learn from each other. Such as the study of computer science and technology is inseparable from the coordination of materials science; the study of artificial intelligence is bound to extend to cognitive science, psychology and brain science. These features make the STA more and more complex which cannot be controlled by a single field. At the same time, the STA are not confined to the development and accumulation of the knowledge of science and technology. It is also very practical.

\subsection{National Mission}

Because of the importance and influence of science and technology, the development of science and technology is gradually paid more attention to. Comrade Deng Xiaoping put forward the judgment "science and technology is the first productivity" according to the trend and current situation of the development of modern science and technology in the reform and opening-up of our country. Since then, technology has become the national mission and the national responsibility. And with the international competition becoming more and more intense, the importance of the STA gets more and more attention of various countries. The Chinese international hi-tech fair which promotes high-tech achievements transformation and the development of high-tech industry is a convincing example. In recent ten years, the number of countries and regions participate in the fair cumulatively increases by $61.5 \%$, the number of foreign government groups increased by nearly 3 times. Customers from a total of more than 80 countries or regions participate in the exhibition, trade and business.

\section{The Characteristics of STP Research}

According to the viewpoint of this paper, the characteristics of STA to a great extent decide the characteristics of STP research. According to the characteristics of the STA summarized above, some of the basic characteristics of STP research can be deduced as follows.

\subsection{Timeliness and Scientificalness of Research}

The influence of STA on human society is wide, fast and irreversible, which makes the existing STP research often lag behind the social impact of STA and not timely. This requires the STP research in the future to react quickly and timely to the effects of STA and predict even without specific influence of STA to do some prevention work for the adverse effects .At the same time, the great impact of STA on human society requires the STP research must be scientific, such as the establishment of STP research methods, the expansion of the research subject of STP, the development of accurate quantitative research, the establishment of STP scientific research paradigm [13].

\subsection{Tolerant and Face up to the Failure of STA}

Because of the market and technology risk of STA, science and technology activities may not always lead to success. To a certain extent, failure is inevitable. This is also the reason for the existence of STP. Professor Su Jun of the school of public administration of Tsinghua University believes that STP exists because of the existing risk of STA. STP need to develop appropriate policies to avoid facing the risks and difficulties in STA. STP research also need the scholars to effectively develop policies to minimize the risk and difficulty. At the same time, STP research needs to tolerate the failure in STA in the concept of value. Because it cannot be completely avoided in the present society. At the same time, the courage to face up to the failure in STA is also needed. The research of STA should initiate the government to help and support the subject of STA to promote STA to carry 
out smoothly.

\subsection{Normative Guidance of STA in the Concept of Value}

Inevitable ethical anomie in the process of STA makes ethical issues become an important content of STP research. At the same time, the hazards brought by ethical anomie require STP research to normatively guide the STA in terms of value and set the appropriate code of ethics and value norms for the STA to guide it to go in the right way.

\subsection{Expand the Research Subject of STP}

The complex characteristics of cross domain, cross discipline and cross level of STA also make STP research not be a simple task and the request of the researchers is higher than the other disciplines. This requires that STP research should not be confined to a certain field and academic attainments. The research subject of STP should be expanded, so that the different research subject can use their own advantages to cooperate with each other. Simultaneously researchers should combine the theory research and practical problems to finish this complicated work.

The subjects of STP include the subject of policy formulation, policy execution and policy evaluation. From a practical point of view, these three aspects all cannot do without the active participation of the people, the government, the academia and even the NGO. Therefore, according to the reality that the "academic" is higher than "practice" in STP research at present in our country, social from all walks should be actively promoted to involve in STP research.

\subsection{Research Should Be National Demand Oriented}

The national mission of STA requires that it must be based on national demand and works for STP decision. This is the summary of the characteristics of US STP by scholars and to a certain extent it can be used for China's STP. The scholars have pointed out, after a careful analysis of the development of STP, you can find factors of science and technology development and innovation. However, a more important factor is the public management reform from the government rather than the scientific community [10]. STP research essentially works for the development of nation and the well-being of the people so both the STA and STP research should firmly bear the values in mind.

\section{Conclusions}

This paper argues that the characteristics of STA influence STP research. The characteristics of the current STA are as below: its effect is of wide range, high speed and irreversible; Facing with the risk of technology and market; Ethical anomie; Interdisciplinary, crossing level and domain; National mission, etc. These characteristics require that the research of STP should have the characteristics as below: Timeliness and scientific; Tolerance and face up to failure; Normative values; Extend research subject; national demand oriented, etc.

This conclusion has a certain theory and practical significance. In theoretical research, the characteristics of STP research from the perspective of the characteristics of STA are a new point of view, a new light on the understanding of STP research. In practice, we suggest that the STP research don't forget the changing characteristics of STA in practice, and adjust continuously to make the research keep pace with the times and bring new breakthrough and contribution to society.

Note that, in fact, in the process of deduction from the characteristics of STA to the characteristics of STP research, the relationship between these two is not complete correspondence. For instance, the fast and irreversible influence of STA also requires STP research to guide STA correctly on values. Because once the values of STA deviate from the mainstream values of society, coupled with the impact of STA, these two will have a great damage to the social stability. Therefore, the corresponding relationship express that the latter is the main result of the former, rather than strictly complete correspondence.

\section{Acknowledgements}

This work is supported by Special Work of Innovation Method: The Key Method, Tool Integration, Demonstra- 
tion and Extension (2013IM010100) and Research on the Intellectual Property Rights Promoting the Construction of Hefei Wuhu Bengbu Independent Innovation Pilot Area (1402052002).

\section{References}

[1] Zhou, H.D. (2011) Science and Technology Policy Studies: Evolution, Differentiation, and Convergence. Science of Science and Management of S. \& T., 11, 5-13.

[2] Zhang, Z.C. (2009) Philosophy of Science and Technology. Nanjing Normal University Press, Nanjing.

[3] He, Y.M. (1999) The Characteristics of the Singapore Science and Technology Activities from the Perspective of R\&D Survey. Global Science, Technology and Economy Outlook, 7, 37.

[4] Yan, G.D. (1999) The Characteristics of Science and Technology Activities of Large and Medium-Sized Industrial Enterprises in Shanghai. East China Science and Technology, 8, 16-17.

[5] Song, Z.F. (1998) Some Characteristic of Contemporary Science and Technology Activities. Science \& Technology Review, 5, 7-10.

[6] Sheng, J.X. and Cheng, L.B. (2002) The Analysis of the Current Situation of Science and Technology Policy Research of China. Forum on Science and Technology in China, 2, 35-39.

[7] Liang, Y.X. and Li, Z.F. (2010) Knowledge Map of the Science and Technology Policy in China Based on CSSCI. Forum on Science and Technology in China, 10, 86-95.

[8] Bao, S. (2008) The Organization Form and the Historical Evolution of Science and Technology Activities. Science and Technology Management Research, 7, 451-453.

[9] Luan, C.J., Hou, H.Y., Hou, J.H. (2009) Cooperative Network of State and the Organization of High Yield on International Science and Technology Policy Research. Science and Technology Management Research, 3, 41-43.

[10] Fan, C.L. and Ma, X.L. (2013) A Study of the Development of Science of Science Policy in U.S and Its Inspiration for China. China Soft Science, 10, 168-181.

[11] Li, F.Q., Ge, X.Q. and He, S.Y. (1998) Knowledge Economy and Knowledge Products. Social Sciences Documentation Publishing House, Beijing.

[12] Su, J. (2014) Public Policy for Science and Technology: An Introduction. Science Press, Beijing.

[13] Du, B.G. (2010) Problems and Countermeasures for the Comparative Study of Science and Technology Policy in China. Scientific Management Research, 4, 59-62. 